\title{
Red-Light Triggered Photouncaging from Dicyanomethylene-4H-py- ran Phototrigger in Aqueous Solution: Applied as a Single Compo- nent Nano-Drug Delivery System for Cancer Therapy
}

\author{
Biswajit Roy, ${ }^{\text {a }}$ Rakesh Mengji, ${ }^{\text {b,c }}$ Moumita Kundu, ${ }^{\text {d }}$ Sujit Kumar Ghosh, ${ }^{\text {a }}$ Mahitosh Mondal, ${ }^{\text {d }}$ Avijit \\ Jana, ${ }^{\mathrm{b}, \mathrm{c}}$ N. D. Pradeep Singh ${ }^{* a}$ \\ aDepartment of Chemistry, Indian Institute of Technology Kharagpur, Kharagpur-721302, India. \\ bDepartment of Organic Synthesis and Process Chemistry, CSIR-Indian Institute of Chemical Technology Hyderabad, Uppal \\ Road, Hyderabad 500007, India. \\ ${ }^{c}$ Academy of Scientific and Innovative Research (AcSIR), Ghaziabad 201002, India.

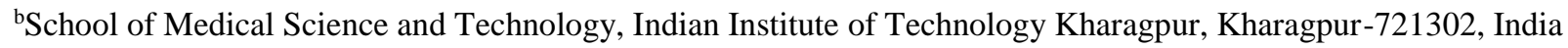

KEYWORDS. Red light responsive, Photocage, NIR Absorbing, Nanoparticles, Anticancer drug release.

\begin{abstract}
The development of Photoremovable protecting groups (PRPGs), which can be activated in the 'phototherapeutic window' for biological applications, is highly challenging. Only PRPGs based on BODIPY dye have been developed so far, which can be excited $\geq 600 \mathrm{~nm}$. Herein, we developed for the first time NIR dye hydroxystyryl dicyanomethylene-4H-pyran (DCM) as a PRPG that can be operated in the phototherapeutic window. Ours easily synthesized DCM photocages efficiently released aromatic and aliphatic carboxylic acids in an aqueous solution on irradiation using the light of wavelength $\geq 600 \mathrm{~nm}$ and $650 \mathrm{~nm}$, separately. As an application, we used our DCM-PRPG as a single component nanocarrier drug delivery system (DDS) to uncage valproic acid (a known histone deacetylase inhibitor) for cancer treatment. In vitro studies revealed that our DDS, hydroxystyryl dicyanomethylene-4H-pyran valproic acid conjugate nanoparticles (DCM-VPA-NPs) exhibited good cellular internalization, biocompatibility, and enhanced cytotoxicity upon irradiation.
\end{abstract}

\section{INTRODUCTION}

Photoremovable Protectecting Groups (PRPGs) operated in the 'phototherapeutic window' $(600-950 \mathrm{~nm})^{1}$ for biological applications are in actual demand. ${ }^{2}$ Last four decades, researchers developed PRPGs that are primarily responsive towards UV and visible light (up to green light). Unfortunately, the light of these regions is less suitable for biological applications ${ }^{3}$ due to (i) strong optical scattering which results in poor tissue penetration (ii) competitive absorbance with natural pigments like hemoglobin (iii) as the light belongs to this region associated with high energy, it results overheating and cytotoxicity. Interestingly, such limitations can be minimized by light $\geq 600 \mathrm{~nm}$ for photouncaging. Since uncaging of bioactive molecules can be carried out using NIR light which is less harmful to the tissues with high penetration ability, they are suitable for drug delivery, gene delivery, neurotransmitter release, etc. ${ }^{3}$ In recent times, few indirect uncaging strategies of bioactive molecules utilizing the light of wavelength $\geq 600 \mathrm{~nm}$ are reported. ${ }^{4}$ For example, aminobenzoquinone photocages were developed, which cyclized upon irradiation with red light, resulting in the release of caged bioactive molecules via a self-immolative pathway. ${ }^{4 a-b}$ Next, Schnermann's group reported an interesting cyaninebased photocages, ${ }^{4 c-d}$ which performed as a photosensitizer to generate singlet oxygen upon irradiation at $690 \mathrm{~nm}$. The produced singlet oxygen breaks the photocage via oxidative cleavage, and subsequent hydrolysis leads to the (a) Prior art: Uncaging from BODIPY-PRPG

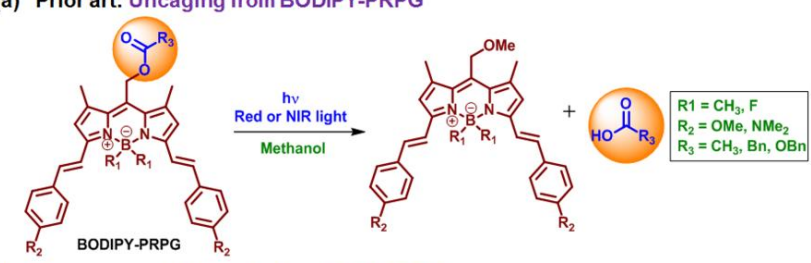

(b) Present work: Uncaging from DCM-PRPG

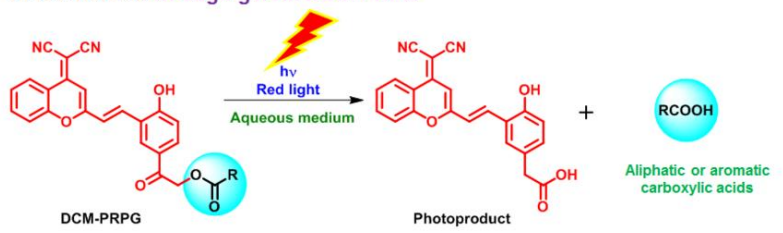

Scheme 1: Photouncaging of active molecules within the 'phototherapeutic window' via single-photon excitation by (a) BODIPY based PRPG in methanol and (b) DCM based PRPG in the aqueous medium.

uncaging of the caged molecule. However, it is always advantageous to use small organic PRPGs that directly released the active molecule with single-photon excitation using red/NIR light as it provides spatio-temporal control over the release. $^{2,3}$

In 2015, Weinstain group 5 and Winter group ${ }^{6}$ independently introduced meso-substituted BODIPY based PRPGs to release carboxylic acids with a green light $(\geq 500 \mathrm{~nm})$. Later, the Winter group developed boron methylated BODIPY photocages 
(Scheme 1a) to release substrates on excitation with Far Red/ NIR light by blocking the unproductive conical intersection. ${ }^{1}$ Feringa's group also developed red light triggered uncaging of amines from meso-substituted BODIPY photocages. ${ }^{7}$ Klan's group reported BODIPY based carbon monoxide-releasing molecules (CORMs) upon irradiation of light within the 'phototherapeutic window' ${ }^{8}$ Recently, Weinstain's group developed water soluble BODIPY photocages having tunable cellular localization. ${ }^{9}$ The exciting aspect of BODIPY-PRPGs is that synthetic modifications can manipulate the photophysical and photochemical properties. ${ }^{10}$ However, most of the BODIPY derivatives are poorly soluble in an aqueous medium, and they required a long synthetic route associated with poor overall yield. Hence, there is a need to develop an easily synthesizable PRPG that can directly release the active molecule in an aqueous medium using light within the 'phototherapeutic window' via single-photon excitation.

In recent times, NIR emissive dyes based on Hydroxystyryl dicyanomethylene-4H-pyran (DCM) chromophore have gained considerable attention, particularly in the area of sensing and imaging of biomolecules, gasotransmitters, enzymes, metal ions, etc. ${ }^{11}$ The exciting feature of DCM derivatives is that they exhibit good absorbance within the phototherapeutic window due to the strong internal charge transfer (ICT) process. ${ }^{11,12}$ Moreover, DCM derivatives are easily synthesizable in a few steps, ${ }^{11,13}$, and exhibit good solubility in the aqueous medium. The above-mentioned interesting properties of DCM derivatives prompted us to develop for the first time DCM chromophore as a red light-activated PRPG.

Herein, we developed an acyl group appended $o$-Hydroxystyryl dicyanomethylene- $4 H$-pyran PRPG to release aliphatic and aromatic carboxylic acids in the 'phototherapeutic window' (Scheme 1b). Our newly developed DCM-PRPG exhibited exciting features like, (i) requires only three steps to synthesize the photocages starting from salicylaldehyde, (ii) good solubility in the aqueous medium (iii) exhibits ground state proton transfer (GSPT) phenomenon in the aqueous medium resulting in the increase in the intensity of absorption and emission ${ }^{14}$ (iv) uncaging of carboxylic can be carried out with red-light (600 - $650 \mathrm{~nm})$ via single-photon excitation.

(a)
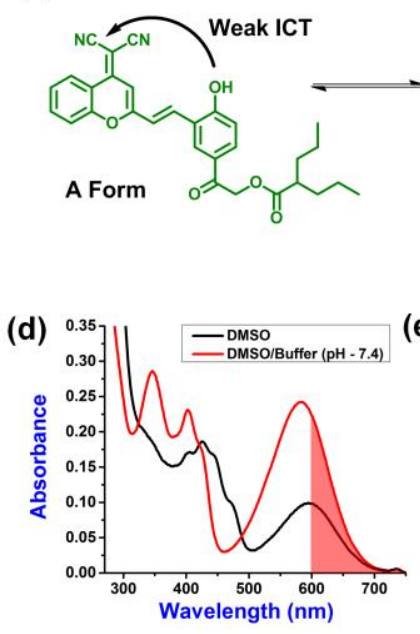

\section{RESULTS AND DISCUSSION}

Synthesis of DCM photocages. We synthesized DCM photocages (5a-d) in three steps starting from salicylaldehyde with 58 to $70 \%$ overall yield (Scheme 2). Friedel-Crafts acylation of salicylaldehyde in the presence of $\mathrm{AlCl}_{3}$ and bromoacetyl bromide produced compound 2 . $^{15}$ Compound $\mathbf{2}$ was then esterified with $p$-anisic acid, $o$-toluic acid, $o$-chlorobenzoic acid individually, in the presence of $\mathrm{K}_{2} \mathrm{CO}_{3}$ in acetonitrile, resulted in compounds 3a-c, respectively. Ester 3d was synthesized from compound $\mathbf{2}$ and sodium valproate in acetonitrile. Next, compound 4 was synthesized by following the literature known procedure, ${ }^{13}$ and finally, condensation reaction between compounds 3a-d and $\mathbf{4}$ produced our final photocages (5a-d), respectively. Experimental details and characterization data of the photocages are provided in the supporting information (pages S5-S23).

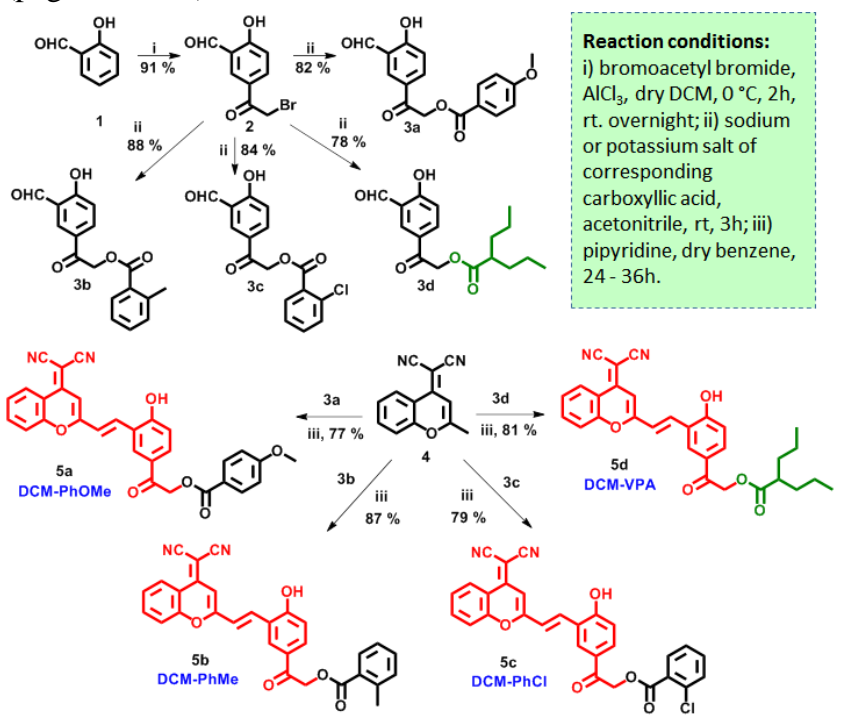

Scheme 2 Synthesis of DCM-photocages (5a-d).

Photophysical properties of DCM photocages. We recorded the UV-vis absorbance and fluorescence of DCM-VPA (5d) as a representative example in different solvents (Figure 1). Figure $1 \mathrm{~b}$ showed two absorption maximum for $\mathbf{5 d}$ due to the existence

Figure 1: (a) Schematic representation of the equilibrium between A-form (enol) and B-form (oxyl) of DCM-VPA. (b) UV-vis absorbance and (c) fluorescence spectra of DCM-VPA $\left(1 \times 10^{-5} \mathrm{M}\right)$ in different solvents. (d) UV-vis absorbance and (e) fluorescent spectra of DCM-VPA (1 x $\left.10^{-5} \mathrm{M}\right)$ in DMSO and DMSO-water $\left(f_{w}=20 \%\right)$ binary mixture. (f) UV-Vis absorbance and (g) fluorescence spectra of DCM-photocages (5a-d) in dry DMSO and 1:1 DMSO/ buffer $(\mathrm{pH}-7.4)$ binary mixture. 
of phenolic structure (A form, Figure 1a) and anionic structure (B form, Figure 1a). The relatively strong absorption peak at around $420 \mathrm{~nm}$ is observed due to the weak internal charge transfer (ICT) generated from the phenolic structure, and the peak around $600 \mathrm{~nm}$ with relatively weak absorption is due to the presence of the anionic form of DCM-VPA 5d, which exhibits strong ICT. ${ }^{12 \mathrm{~b}}$ Interestingly, in the emission spectra, we also observed two emission maximum for DCM-VPA 5d at around $520 \mathrm{~nm}$ and $730 \mathrm{~nm}$ (Figure 1c).

We noticed an interesting phenomenon for DCM-VPA 5d in the aqueous medium (DMSO-water binary mixture), the intensity of the peaks having an absorption maximum at $600 \mathrm{~nm}$ (Figure 1d) and emission maximum at $730 \mathrm{~nm}$ (Figure 1e) increased, which can be attributed due to the increase in the population of the anionic form (B form, Figure 1a). This phenomenon is known as a ground-state proton-transfer (GSPT) process, where solvent molecules assist the proton transfer, resulting in the redshift of absorption and emission maximum due to the formation of an anionic structure. ${ }^{14}$

To confirm that our photocages are GSPT active, we recorded the absorption (Figure 1f) and emission spectra (Figure 1g) of all the photocages (5a-d) in dry DMSO and 1:1 DMSO: buffer (pH 7.4) mixture ${ }^{14}$. We noted that the addition of buffer solution enhanced the intensity of the absorption and the emission maximum of our photocages by ten folds with a slight blue-shift $(\sim 20 \mathrm{~nm})$ of the absorption maximum $(580 \mathrm{~nm})$. We calculated the fluorescent quantum yield of our photocages (Table 1) in a 1:1 DMSO/buffer ( $\mathrm{pH} 7.4$ ) binary mixture using rhodamine $\mathrm{B}$ $\left(\phi_{\mathrm{F}}=0.31\right.$ in water) as the standard. ${ }^{17}$

Table 1: Overall synthetic yields and photophysical properties of DCMphotocages (5a-d):

\begin{tabular}{|c|c|c|c|c|c|c|}
\hline \multirow[b]{2}{*}{ Photocages } & \multirow{2}{*}{$\begin{array}{l}\text { Caged } \\
\text { carboxylic } \\
\text { acids }\end{array}$} & \multirow[b]{2}{*}{$\begin{array}{l}\text { Yield } \\
(\%)^{\mathrm{a}}\end{array}$} & \multicolumn{2}{|c|}{ Absorption } & \multicolumn{2}{|c|}{ Emission } \\
\hline & & & $\begin{array}{l}\lambda_{\max }^{\mathrm{b}} \\
(\mathrm{nm})\end{array}$ & $\varepsilon^{\mathrm{c}}$ & $\begin{array}{l}\lambda_{\max }{ }^{\mathrm{d}} \\
(\mathrm{nm})\end{array}$ & $\left(\phi_{\mathrm{F}}\right)^{\mathrm{e}}$ \\
\hline $\begin{array}{c}\text { DCM- } \\
\text { PhOMe } \\
\mathbf{5 a}\end{array}$ & & 58 & 576 & 54900 & 744 & 0.39 \\
\hline $\begin{array}{c}\text { DCM-PhMe } \\
\mathbf{5 b}\end{array}$ & & 70 & 578 & 78300 & 743 & 0.4 \\
\hline $\begin{array}{c}\mathrm{DCM}-\mathrm{PhCl} \\
\mathbf{5 c}\end{array}$ & & 61 & 574 & 68700 & 740 & 0.46 \\
\hline $\begin{array}{c}\text { DCM-VPA } \\
\mathbf{5 d}\end{array}$ & & 58 & 580 & 60800 & 750 & 0.37 \\
\hline
\end{tabular}

${ }^{a}$ Based on the isolated yield after three steps. ${ }^{b}$ Maximum absorption wavelength. ${ }^{\mathrm{c}}$ Molar absorption coefficient $\boldsymbol{\varepsilon}\left(\mathrm{M}^{-1} \mathrm{~cm}^{-1}\right)$. ${ }^{\mathrm{d}}$ Maximum emission wavelength. ${ }^{\mathrm{e}}$ Fluorescence quantum yield $\left(\phi_{\mathrm{F}}\right)$.

Stability of DCM photocages. We also examined the stability of the photocages (5a-d) in biological $\mathrm{pH}$ and two different $\mathrm{pH}$ solutions (5.5 and 8) under the dark condition at $37^{\circ} \mathrm{C}$. Table S1 indicated that the decomposition of photocages was less than $5 \%$ after seven days.

Photouncaging of carboxylic acids from DCM photocages. Next, we investigated the photouncaging ability of photocages 5a-d by one-photon irradiation using the light of 600 and 650 $\mathrm{nm}$ wavelengths, separately. We prepared a $4 \mathrm{ml}\left(1 \times 10^{-4} \mathrm{M}\right)$ solution of photocages in DMSO/PBS buffer (pH-7.4) binary mixture with $75 \%$ water fraction $\left(f_{\mathrm{w}}\right)$ in a cuvette and exposed to a $150 \mathrm{~W}$ xenon arc lamp as the light source assembled with a band-pass filter at $600 \pm 10 \mathrm{~nm}$, separately. The uncaging process was monitored by reverse-phase high-performance liquid chromatography (RP-HPLC), mass spectroscopy, absorption, and emission spectroscopy (Figure 2 \& Figure S20S26). We observed that DCM-photocages released the caged carboxylic acids in high chemical (90-95\%) and moderate quantum yields (Table 2). The quantum yield for the photorelease was calculated by employing potassium ferrioxalate as an actinometer. ${ }^{1,15,17}$

As a representative example, the photorelease of DCM-VPA 5d was monitored by RP-HPLC at different irradiation time intervals. The RP-HPLC chromatograms (Figure 2a) of DCM-
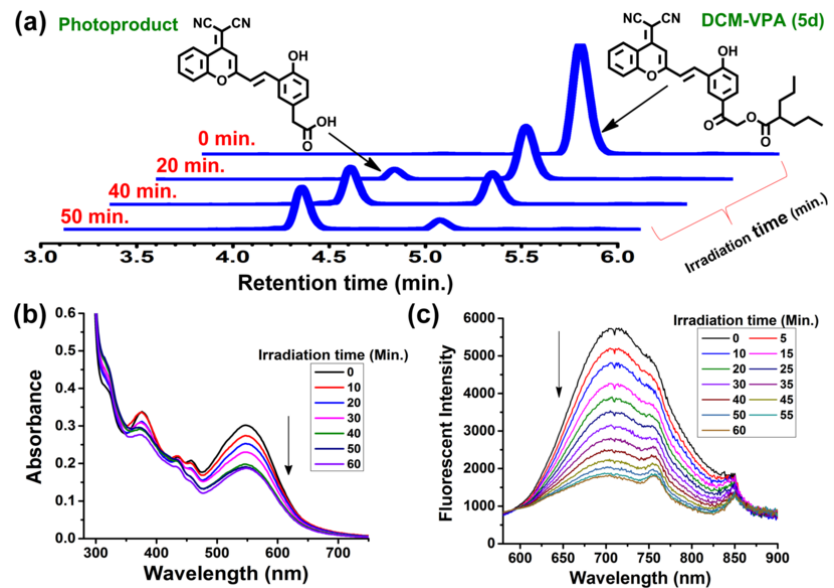

(c)

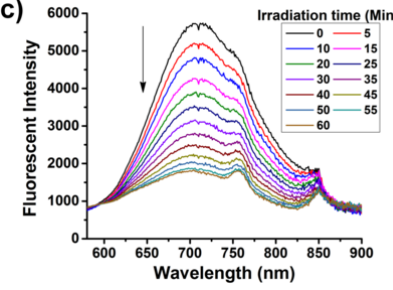

Figure 2: (a) HPLC overlay chromatogram of DCM-VPA 5d at different time intervals of light irradiation $(600 \pm 10 \mathrm{~nm})$; Change in the (b) absorbance and (c) fluorescent spectral profile with increasing irradiation time (irradiation wavelength $=600 \pm 10 \mathrm{~nm}$ ).

VPA 5d showed that the initial peak at $t_{R}=5$ min gradually decreases with increasing irradiation time, indicating the photodecomposition of DCM-VPA. On the other hand, a concomitant increase of a new peak at $t_{R}=4.2$ min was noted, which indicated the formation of the photoproduct (Scheme 1b). The formation of the photoproduct and the released valproic acid was confirmed by HRMS (Figure S24-S26).

Next, we quantified the photodecomposition of DCM-VPA from the HPLC chromatograms (Figure 3a). Figure 3a showed that $\geq 90 \%$ of photouncaging occurred after $55 \mathrm{~min}$ of irradiation. We also observed notable changes in the absorption (Figure 2b) and emission spectra (Figure 2c) of DCM-VPA during photolysis at $600 \pm 10 \mathrm{~nm}$ for $55 \mathrm{~min}$. The decrease in the intensity of absorption and emission maxima can be attributed to the loss of conjugation of hydroxyl with the phenacyl group due to the formation of photo-Favorskii rearranged product ${ }^{3,15,18}$ (Scheme 1b). Initially, the hydroxyl group of the photocage participated in cross-conjugation with the phenacyl and DCM groups; therefore, the hydroxyl proton becomes more acidic and quickly gets deprotonated to generate oxyl anion resulting in strong red fluorescence. However, the hydroxyl proton becomes less acidic after photolysis because the hydroxyl group is only conjugated to the DCM moiety and exhibited weak red fluorescence. Therefore, uncaging of carboxylic acids from DCM-photocages can be easily monitored by decrease in fluorescence intensity. Similarly, we carried out the photolysis of DCM-PhOMe 5a, DCM-PhMe 5b, DCM-PhCl 5c at $600 \pm 10$ $\mathrm{nm}$. We quantified the photodecomposition amount of these 
photocages from the peak area of HPLC chromatograms for the individual photocages (Figure 3a). We also observed a notable decrease in the fluorescence intensity of these photocages during photolysis (Figure S20).

We also checked the uncaging ability of all the photocages (5ad) in a similar procedure at $650 \mathrm{~nm}$ (band-pass filter at $650 \pm$ $10 \mathrm{~nm}$ ). We noted that the time required for $90 \%$ uncaging of carboxylic acids was longer compared to the photouncaging at $600 \mathrm{~nm}$ (Table $2 \&$ Figure S21). For example, the time required for $90 \%$ photodecomposition of DCM-VPA at $600 \mathrm{~nm}$ is $55 \mathrm{~min}$ (Figure 3a). At $650 \mathrm{~nm}$, it required $140 \mathrm{~min}$ of irradiation (Table 2, Figure S22). We have tabulated the photochemical quantum yields of DCM-photocages at $600 \mathrm{~nm}$ and $650 \mathrm{~nm}$ in Table 2.

Table 2: Photochemical properties of DCM-photocages (5a-d):

\begin{tabular}{|c|c|c|c|c|c|}
\hline \multirow[b]{2}{*}{ Photocages } & \multirow[b]{2}{*}{$\begin{array}{l}\text { Carboxylic } \\
\text { acid (pKa) }\end{array}$} & \multicolumn{2}{|c|}{ Irradiation at $600 \mathrm{~nm}$} & \multicolumn{2}{|c|}{ Irradiation at $650 \mathrm{~nm}$} \\
\hline & & $\begin{array}{c}\text { Time } \\
\text { required } \\
\text { for } 90 \% \\
\text { uncaging }^{\text {a }}\end{array}$ & $\begin{array}{l}\text { Uncaging } \\
\text { Quantum } \\
\text { yield } \Phi_{\mathrm{u}}{ }^{\mathrm{b}}\end{array}$ & $\begin{array}{c}\text { Time } \\
\text { Required } \\
\text { for } 90 \% \\
\text { uncaging }^{\mathrm{a}}\end{array}$ & $\begin{array}{l}\text { Uncaging } \\
\text { Quantum } \\
\text { yield } \Phi_{\mathrm{u}}{ }^{\mathrm{b}}\end{array}$ \\
\hline $\begin{array}{c}\text { DCM- } \\
\text { PhOMe } \\
\mathbf{5 a}\end{array}$ & $\begin{array}{c}o \text {-Anisic } \\
\text { acid } \\
(4.47)\end{array}$ & 68 & 0.068 & 185 & 0.026 \\
\hline $\begin{array}{c}\text { DCM- } \\
\text { PhMe } \\
\mathbf{5 b}\end{array}$ & $\begin{array}{c}o \text {-Toluic } \\
\text { acid } \\
(3.96)\end{array}$ & 49 & 0.093 & 128 & 0.036 \\
\hline $\begin{array}{c}\text { DCM- } \\
\mathrm{PhCl} \\
\mathbf{5 c}\end{array}$ & $\begin{array}{c}o \text {-Chloro } \\
\text { benzoic } \\
\text { acid } \\
(2.89)\end{array}$ & 35 & 0.13 & 105 & 0.051 \\
\hline $\begin{array}{c}\text { DCM- } \\
\text { VPA } \\
\mathbf{5 d}\end{array}$ & $\begin{array}{c}\text { Valproic } \\
\text { acid } \\
(4.8)\end{array}$ & 55 & 0.084 & 140 & 0.032 \\
\hline
\end{tabular}

${ }^{\mathrm{a}} 90 \%$ release of carboxylic acids was determined from the HPLC peak area with respect to irradiation time in minutes. The photochemical quantum yield $\left(\phi_{\mathrm{u}}\right)$ of DCM-photocages was calculated using potassium ferrioxalate as an actinometer. ${ }^{17}$

The results indicated that our DCM PRPG efficiently uncaged carboxylic acid in the phototherapeutic window.

To confirm the precise control of uncaging is entirely dependent upon the incident light, we carried out a simple light turn on-off experiment. ${ }^{19}$ In which, we exposed the DCM-VPA 5d solution to light and dark conditions at different intervals. Figure $3 b$ clearly showed that in dark conditions, no release was observed, indicating light is solely responsible for the release of valproic acid.
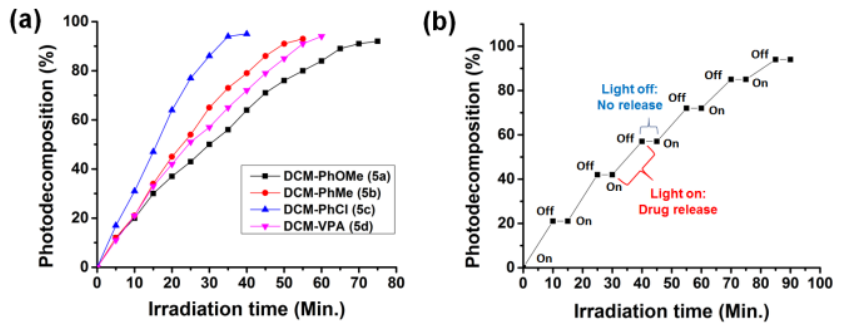

Figure 3: (a) The amount of uncaging from DCM-photocages (5a-5d) on photolysis $(600 \pm 10 \mathrm{~nm})$ at different time intervals. (b) Photouncaging of DCM-VPA 5d under light and dark conditions. "On" and "Off" imply switching on and off of the light source, respectively.

Mechanism of photorelease from DCM photocages. Next, we investigate the photouncaging mechanism of DCM photocages. As our PRPG is a derivative of p-hydroxyphenacyl
PRPG, we believed that the photouncaging occurred via PhotoFavorskii rearrangement from the triplet excited state. To check whether the photouncaging happens from the triplet excited state, we performed two different experiments. At first, the photolysis of DCM-VPA was carried out in the presence of a triplet quencher potassium sorbate $(\mathrm{PoS}) .{ }^{15} \mathrm{We}$ found that in the presence of PoS the drug release almost got quenched (Figure S27). Secondly, we examine the photolysis of DCM-VPA in the presence and absence of aerated oxygen. Interestingly, the photochemical quantum yield of DCM-VPA was found to be higher in the case of nitrogen-degassed solution $\left(\Phi_{\mathrm{u}}=0.084\right)$

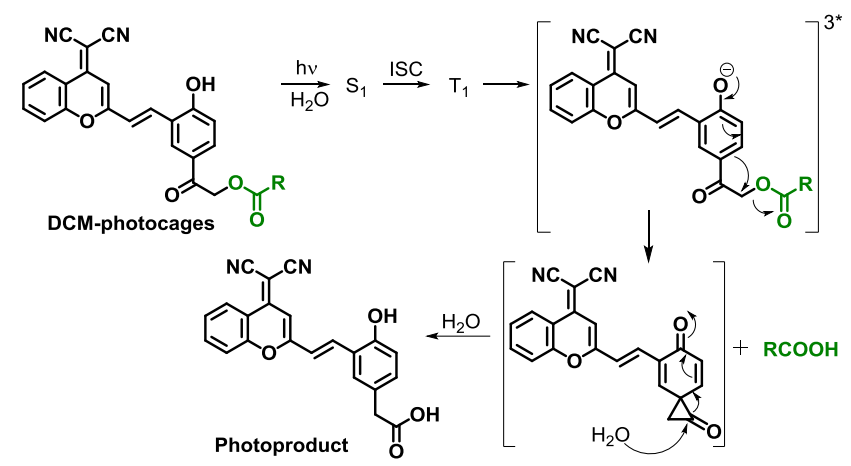

Scheme 3: Possible photorelease mechanism of the DCM-photocages.

than in aerated solution $\left(\Phi_{\mathrm{u}}=0.067\right)$. This decrease in the photochemical quantum yield of DCM-VPA in aerated solution is due to the possible energy transfer from the triplet excited state of DCM-VPA to oxygen, which perturbs the photodecomposition.

Therefore, based on the literature reports ${ }^{3,15,18}$ and our experimental findings, we proposed the possible photorelease mechanism of our DCM-PRPG (Scheme 3). At first, the DCM photocage gets excited to its singlet state in the presence of water, and then it undergoes efficient intersystem crossing (ISC) to its triplet excited state. From the triplet excited state, the leaving group departs along with the formation of a putative spirodione intermediate. The spirodione intermediate upon hydrolytic ringopening gives the photoproduct (Scheme 3).

In vitro applicability of DCM-VPA as nano DDS. The positive outcome of red light triggered uncaging of carboxylic acids encouraged us to investigate the intracellular drug release ability of our PRPG. Since, one of our photocages, DCM-VPA, was attached with valproic acid which is an anticancer drug (a histone deacetylase inhibitor that decondensed the chromatin and prohibited cell proliferation ${ }^{20}$ ), we used cancerous cell lines for in vitro drug release.

At first, we prepared nanoparticles of DCM-VPA by reprecipitation technique for biological application by following our previously reported procedure (Figure 4a). ${ }^{21}$ We took the transmission electron microscopy (TEM) images to understand the shape and size of the formed nanoparticles (NPs). From Figure $4 \mathrm{~b}$ and $4 \mathrm{c}$, we observed that the average size of the DCM-VPANPs is around $\sim 7 \mathrm{~nm}$ with almost spherical in shape.

Next, the cellular internalization of DCM-VPA-NPs were monitored by confocal laser scanning microscopy (CLSM). B16F10 cells were incubated with DCM-VPA-NPs (Figure 5a), and the cell nuclei were stained with 4,6-diamidino-2-phenylindole (DAPI). From Figure 5b (i-iii), we noticed that DCM-VPA-NPs got internalized after $4 \mathrm{~h}$ of incubation and exhibited red fluorescence. Next, we irradiated these pretreated cells for $60 \mathrm{~min}$ and observed a decrease in the fluorescent intensity [Figure $5 \mathrm{c}$ 
(i-iii)]. The change in the fluorescent intensity roughly indicates cytosolic drug release by DCM-VPA-NPs.

Finally, the cytotoxicity of DCM-VPA-NPs was examined by MTT assay [MTT= 3-(4,5-dimethylthiazol-2-yl)-2,5-diphenyltetrazolium bromide, a yellow tetrazole] using MDA-MB-231 cell line. We observed $>80 \%$ cell viability at different concentrations of DCM-VPA-NPs after 72h incubation period (Figure 6). Next, we irradiated DCM-VPA-NPs pretreated cells and compared the cytotoxicity with sodium valproate treated cells after light irradiation (Figure 6). The results indicated that DCM-VPA-NPs $\mathbf{5 d}$ are showing enhanced cytotoxicity upon irradiation compared to free sodium valproate. The enhanced cytotoxicity can be attributed to the intracellular drug release. Therefore, our PRPG is efficient in releasing caged active molecules within the cells upon red light irradiation. (a)

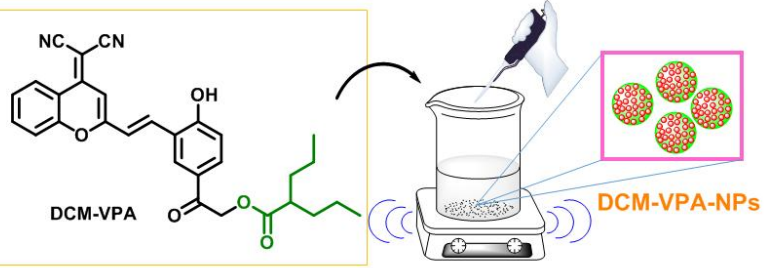

(b)

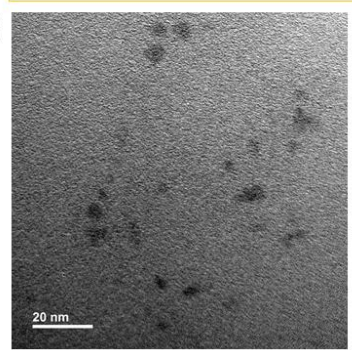

(c)

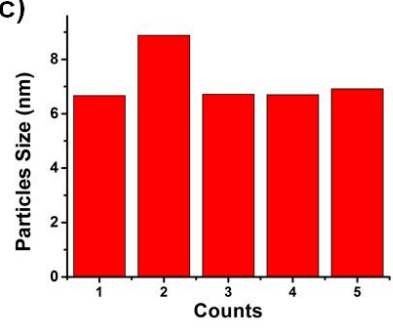

Figure 4: (a) Schematic representation of the preparation of DCM-VPANPs; (b) TEM image of DCM-VPA-NPs; (c) Average size of the DCMVPA-NPs.

(a)

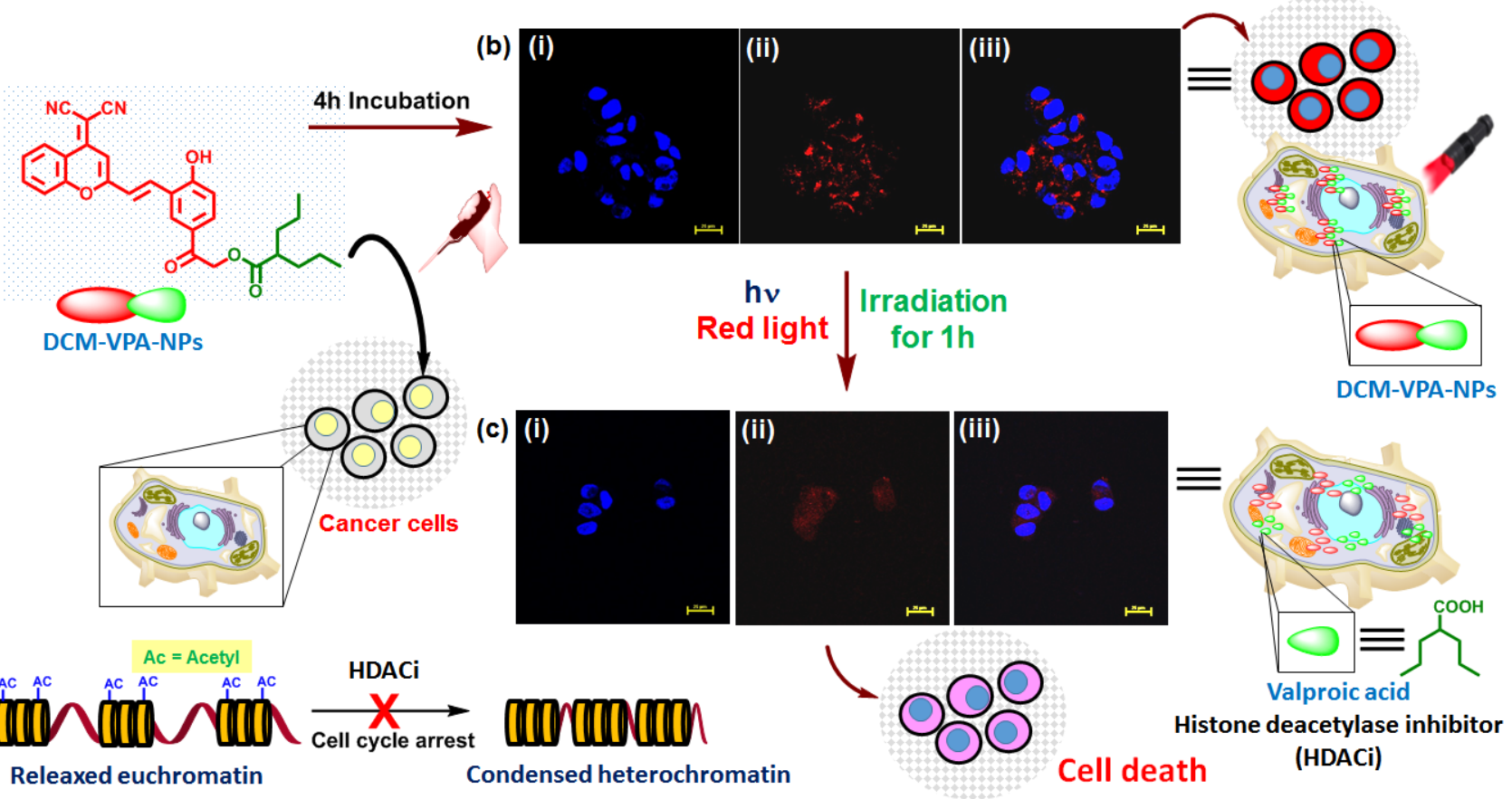

Figure 5: (a) Schematic representation of the preparation of DCM-VPA-NPs; Live B16F10 cell lines treated with DCM-VPA-NPs and DAPI and CLSM images were collected after $4 \mathrm{~h}$ of incubation period via [b(i)] blue channel, [b(ii)] red channel, [b(iii)] merged image of blue \& red channel; B16F10 cell lines treated with DCM-VPA-NPs and DAPI were irradiated for 1h and CLSM images were collected via, [c(iv)] blue channel, [c(ii)] red channel, [c(iii)] merged image of blue \& red channel; (d) Schematics of histone deacetylase inhibitory activity of valproic acid.

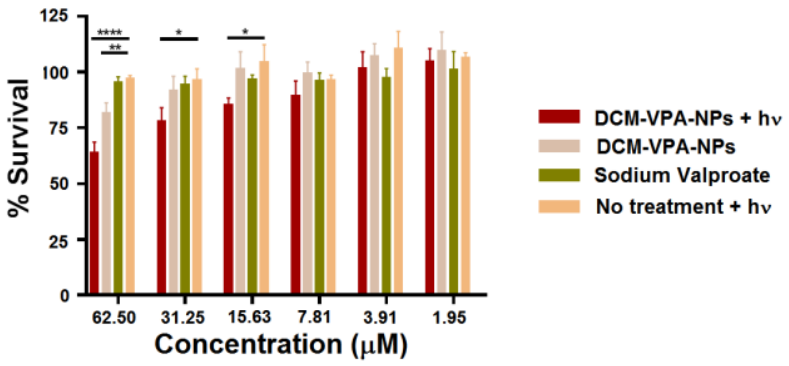

Figure 6: Cell viability assays for DCM-VPA-NPs and sodium valproate with MDA-MB-231 cell line. Data presented are the average of the data obtained in triplicate independent experiments and presented relative to a control, and the results were presented as mean \pm SEM. The statistical analysis was done using one-way ANOVA. $\mathrm{p}$-Value $<0.05$ was considered statistically significant. *, p < 0.05; **, p < 0.01; ***, p < 0.001;***, p < 0.0001 .

\section{CONCLUSION}

In conclusion, we developed NIR dye DCM as a PRPG for carboxylic acids with excellent uncaging efficiency at a higher wavelength (above $600 \mathrm{~nm}$ ). Our DCM-photocages (5a-d) are synthesized by three steps starting from commercially available salicylaldehyde with a good overall yield. Our DCMphotocages exhibited the GSPT phenomenon in the aqueous medium, resulting in increased absorption and emission intensity. DCM-PRPG released efficiently carboxylic acids based on p-hydroxyphenacyl mechanism. Further, we used our DCMPRPG to develop a single component nanocarrier drug delivery system (DDS) to uncage valproic acid for cancer treatment. The positive aspect of our single component nanocarrier DDS is that it does not require any inorganic components. 
In vitro studies revealed that our nano-DDS, DCM-VPA-NPs exhibited good cellular internalization, biocompatibility, and enhanced cytotoxicity upon irradiation. In the future, we want to modify the DCM-PRPG for uncaging molecules having different functional groups at a higher wavelength NIR light (above $700 \mathrm{~nm}$ ).

\section{ACKNOWLEDGMENT}

We thank DST SERB (Grant No. EMR/2016/005885 and SERB/F/6429/2020-21) for financial support. The CSIR-IICT Com-munication number for this manuscript is IICT/Pubs./2021/196.

\section{REFERENCES}

(1) Peterson, J. A.; Wijesooriya, C.; Gehrmann, E. J.; Mahoney, K. M.; Goswami, P. P.; Albright, T. R.; Syed, A.; Dutton, A. S.; Smith, E. A.; Winter, A. H. Family of BODIPY Photocages Cleaved by Single Photons of Visible/Near-Infrared Light. J. Am. Chem. Soc., 2018, 140, 7343-7346

(2) Weinstain, R.; Slanina, T.; Kand, D.; Klán, P. Visible-to-NIRLight Activated Release: From Small Molecules to Nanomaterials. Chem. Rev., 2020, 120, 13135-13272.

(3) Klán, P.; Šolomek, T.; Bochet, C. G.; Blanc, A.; Givens, R.; Rubina, M.; Popik, V.; Kostikov, A.; Wirz, J. Photoremovable Protecting Groups in Chemistry and Biology: Reaction Mechanisms and Efficacy. Chem. Rev., 2012, 113, 119-191

(4) (a) Wang, X.; Kalow, J. A. Rapid Aqueous Photouncaging by Red Light. Org. Lett., 2018, 20, 1716-1719. (b) Carling, C.-J.; Olejniczak, J.; Foucault-Collet, A.; Collet, G.; Viger, M. L.; Nguyen Huu, V. A.; Duggan, B. M.; Almutairi, A. Efficient Red Light PhotoUncaging of Active Molecules in Water upon Assembly into Nanoparticles. Chem. Sci., 2016, 7, 2392-2398. (c) Gorka, A. P.; Nani, R. R.; Zhu, J.; Mackem, S.; Schnermann, M. J. A Near-IR Uncaging Strategy Based on Cyanine Photochemistry. J. Am. Chem. Soc., 2014, 136, 14153-14159. (d) Nani, R. R.; Gorka, A. P.; Nagaya, T.; Kobayashi, H.; Schnermann, M. J. Near-IR Light-Mediated Cleavage of AntibodyDrug Conjugates Using Cyanine Photocages. Angew. Chem. Int. Ed., 2015, 54, 13635-13638. (e) Anderson, E. D.; Gorka, A. P.; Schnermann, M. J. Near-Infrared Uncaging or Photosensitizing Dictated by Oxygen Tension. Nat Commun, 2016, 7, DOI 10.1038/ncomms13378. (f) Vorobev, A. Yu.; Moskalensky, A. E. Long-Wavelength Photoremovable Protecting Groups: On the Way to in Vivo Application. Comput. Struct. Biotechnol. J., 2020, 18, $27-34$.

(5) Rubinstein, N.; Liu, P.; Miller, E. W.; Weinstain, R. MesoMethylhydroxy BODIPY: A Scaffold for Photo-Labile Protecting Groups. Chem. Commun., 2015, 51, 6369-6372.

(6) Goswami, P. P.; Syed, A.; Beck, C. L.; Albright, T. R.; Mahoney, K. M.; Unash, R.; Smith, E. A.; Winter, A. H. BODIPY-Derived Photoremovable Protecting Groups Unmasked with Green Light. $J$. Am. Chem. Soc., 2015, 137, 3783-3786.

(7) Sitkowska, K.; Hoes, M. F.; Lerch, M. M.; Lameijer, L. N.; van der Meer, P.; Szymański, W.; Feringa, B. L. Red-Light-Sensitive BODIPY Photoprotecting Groups for Amines and Their Biological Application in Controlling Heart Rhythm. Chem. Commun., 2020, 56 , $5480-5483$

(8) Palao, E.; Slanina, T.; Muchová, L.; Šolomek, T.; Vítek, L.; Klán, P. Transition-Metal-Free CO-Releasing BODIPY Derivatives Activatable by Visible to NIR Light as Promising Bioactive Molecules. J. Am. Chem. Soc., 2015, 138, 126-133.

(9) Kand, D.; Liu, P.; Navarro, M. X.; Fischer, L. J.; Rousso-Noori, L.; Friedmann-Morvinski, D.; Winter, A. H.; Miller, E. W.; Weinstain, R. Water-Soluble BODIPY Photocages with Tunable Cellular Localization. J. Am. Chem. Soc., 2020, 142, 4970-4974.

(10) Slanina, T.; Shrestha, P.; Palao, E.; Kand, D.; Peterson, J. A.; Dutton, A. S.; Rubinstein, N.; Weinstain, R.; Winter, A. H.; Klán, P. In Search of the Perfect Photocage: Structure-Reactivity Relationships in
Meso-Methyl BODIPY Photoremovable Protecting Groups. J. Am. Chem. Soc., 2017, 139, 15168-15175.

(11) (a) Zhang, W.; Huo, F.; Yin, C. Recent Advances of DicyanoBased Materials in Biology and Medicine. J. Mater. Chem. B, 2018, 6, 6919-6929. (b) Li, Z.; Xu, Y.; Xu, H.; Cui, M.; Liu, T.; Ren, X.; Sun, J.; Deng, D.; Gu, Y.; Wang, P. A Dicyanomethylene-4H-Pyran-Based Fluorescence Probe with High Selectivity and Sensitivity for Detecting Copper (II) and Its Bioimaging in Living Cells and Tissue. Spectrochim. Acta A Mol. Biomol. Spectrosc., 2021, 244, 118819.

(12) (a) Wang, B.-B.; Wang, Y.; Wu, W.-N.; Xu, Z.-H.; Zhao, X.L.; Xu, Z.-Q.; Fan, Y.-C. A Near-Infrared Colorimetric and Fluorescent Dual-Channel Probe for Cyanide Detection Based on Dicyanomethylene-4H-Pyran. Inorg. Chem. Commun., 2020, 122, 108245. (b) Yang, J.; Li, M.; Zhu, W.-H. Dicyanomethylene-4H-Pyran-Based NIR Fluorescent Ratiometric Chemosensor for $\mathrm{PH}$ Measurement. Res. Chem. Intermed., 2018, 44, 3959-3969. (c) Rajasekhar, K.; Achar, C. J.; Govindaraju, T. A Red-NIR Emissive Probe for the Selective Detection of Albumin in Urine Samples and Live Cells. Org. Biomol. Chem., 2017, $15,1584-1588$

(13) Wang, J.; Li, B.; Zhao, W.; Zhang, X.; Luo, X.; Corkins, M. E.; Cole, S. L.; Wang, C.; Xiao, Y.; Bi, X.; Pang, Y.; McElroy, C. A.; Bird, A. J.; Dong, Y. Two-Photon Near Infrared Fluorescent Turn-On Probe Toward Cysteine and Its Imaging Applications. ACS Sens., 2016, 1, 882-887.

(14) Roy, B.; Roy, S.; Kundu, M.; Maji, S.; Pal, B.; Mandal, M.; Singh, N. D. P. Ground-State Proton-Transfer (GSPT)-Assisted Enhanced Two-Photon Uncaging from a Binol-Based AIE-Fluorogenic Phototrigger. Org. Lett., 2021, 23, 2308-2313.

(15) Barman, S.; Mukhopadhyay, S. K.; Biswas, S.; Nandi, S.; Gangopadhyay, M.; Dey, S.; Anoop, A.; Pradeep Singh, N. D. A P-Hydroxyphenacyl-Benzothiazole-Chlorambucil Conjugate as a Real-TimeMonitoring Drug-Delivery System Assisted by Excited-State Intramolecular Proton Transfer. Angew. Chem. Int. Ed., 2016, 55,

4194-4198.

(16) Ryan, E. T.; Xiang, T.; Johnston, K. P.; Fox, M. A. Absorption and Fluorescence Studies of Acridine in Subcritical and Supercritical Water. J. Phys. Chem. A, 1997, 101, 1827-1835.

(17) (a) Hatchard, C. G.; Parker, C. A. A New Sensitive Chemical Actinometer - II. Potassium Ferrioxalate as a Standard Chemical Actinometer. Proc. R. Soc. Lond. A, 1956, 235, 518-536. (b) Mandal, S. Ghatak, C.; Rao, V. G.; Ghosh, S.; Sarkar, N. Pluronic Micellar Aggregates Loaded with Gold Nanoparticles (Au NPs) and Fluorescent Dyes: A Study of Controlled Nanometal Surface Energy Transfer. J. Phys. Chem. C, 2012, 116, 5585-5597.

(18) Givens, R. S.; Heger, D.; Hellrung, B.; Kamdzhilov, Y.; Mac, M.; Conrad, P. G.; Cope, E.; Lee, J. I.; Mata-Segreda, J. F.; Schowen, R. L.; et al. The Photo-Favorskii Reaction Ofp-Hydroxyphenacyl Compounds Is Initiated by Water-Assisted, Adiabatic Extrusion of a Triplet Biradical. J. Am. Chem. Soc., 2008, 130, 3307-3309.

(19) Roy, B.; Kundu, M.; Singh, A. K.; Singha, T.; Bhattacharya, S.; Datta, P. K.; Mandal, M.; Singh, N. D. P. Stepwise Dual Stimuli Triggered Dual Drug Release by a Single Naphthalene Based Two-Photon Chromophore to Reverse MDR for Alkylating Agents with Dual Surveillance in Uncaging Steps. Chem. Commun., 2019, 55, 13140-13143.

(20) Blaheta, R. A.; Michaelis, M.; Driever, P. H.; Cinatl, J. Evolving Anticancer Drug Valproic Acid: Insights into the Mechanism and Clinical Studies. Med. Res. Rev., 2005, 25, 383-397.

(21) Jana, A.; Devi, K. S. P.; Maiti, T. K.; Singh, N. D. P. Perylene3-Ylmethanol: Fluorescent Organic Nanoparticles as a Single-Component Photoresponsive Nanocarrier with Real-Time Monitoring of Anticancer Drug Release. J. Am. Chem. Soc., 2012, 134, 7656-7659. 